\title{
Characterization of a synthetic bacterial self- destruction device for programmed cell death and for recombinant proteins release
}

\author{
Lorenzo Pasotti ${ }^{1,2}$, Susanna Zucca ${ }^{1,2}$, Manuel Lupotto ${ }^{2}$, Maria Gabriella Cusella De Angelis ${ }^{2}$ and Paolo Magni ${ }^{1,2^{*}}$
}

\begin{abstract}
Background: Bacterial cell lysis is a widely studied mechanism that can be achieved through the intracellular expression of phage native lytic proteins. This mechanism can be exploited for programmed cell death and for gentle cell disruption to release recombinant proteins when in vivo secretion is not feasible. Several genetic parts for cell lysis have been developed and their quantitative characterization is an essential step to enable the engineering of synthetic lytic systems with predictable behavior.

Results: Here, a BioBrickTM lysis device present in the Registry of Standard Biological Parts has been quantitatively characterized. Its activity has been measured in E. coli by assembling the device under the control of a well characterized N-3-oxohexanoyl-L-homoserine lactone (HSL) -inducible promoter and the transfer function, lysis dynamics, protein release capability and genotypic and phenotypic stability of the device have been evaluated. Finally, its modularity was tested by assembling the device to a different inducible promoter, which can be triggered by heat induction.

Conclusions: The studied device is suitable for recombinant protein release as $96 \%$ of the total amount of the intracellular proteins was successfully released into the medium. Furthermore, it has been shown that the device can be assembled to different input devices to trigger cell lysis in response to a user-defined signal. For this reason, this lysis device can be a useful tool for the rational design and construction of complex synthetic biological systems composed by biological parts with known and well characterized function. Conversely, the onset of mutants makes this device unsuitable for the programmed cell death of a bacterial population.
\end{abstract}

\section{Background}

Naturally occurring lytic and temperate bacteriophages have the ability to provoke the host cell lysis through the expression of specific proteins during the lytic cycle. In many phages, like the T4 phage and the lambda phage, these proteins have been identified and widely studied [1-4]. In particular, holins form stable and nonspecific lesions in the cytoplasmic membrane that allow the lysozymes to gain access to the peptidoglycan layer. Lysozymes are generally soluble proteins with one or more muralytic activities against the three different types of covalent bonds (glycosidic, amide, and peptide) of the peptidoglycan polymer of the cell wall $[5,6]$. The

\footnotetext{
*Correspondence: paolo.magni@unipv.it

'Dipartimento di Informatica e Sistemistica, Università degli Studi di Pavia,

via Ferrata 1, Pavia, Italy

Full list of author information is available at the end of the article
}

combined work of holin and lysozyme results in the degradation of the two cell membranes of gram-negative bacteria, thus causing cell lysis. Antiholin is a third protein involved in this process as it inhibits holin and is responsible for the regulation of its activity [7].

The described lytic mechanism can be exploited for the release of useful recombinant proteins which cannot be secreted by the engineered host strain [8].

Escherichia coli is a widely used organism for recombinant protein production, but its secretion capabilities are limited and recombinant protein targeting to the growth medium has shown to work only with a small set of proteins [9]. For this reason, cell disruption techniques are required to gain the intracellularly expressed protein of interest. Mechanical techniques, such as cell ultrasonication, usually result in protein denaturation caused by the heat produced during the process and
C Biomed Central

() 2011 Pasotti et al; licensee BioMed Central Ltd. This is an Open Access article distributed under the terms of the Creative Commons Attribution License (http://creativecommons.org/licenses/by/2.0), which permits unrestricted use, distribution, and reproduction in any medium, provided the original work is properly cited. 
some of them are also unfeasible on industrial scale, whereas non-mechanical techniques, such as chemical membrane degradation with detergents or enzymes, involve the purchase of expensive reagents [8]. Other recently proposed mechanical, chemical, physical and enzymatic treatments to disrupt the cell membrane, especially focused on high throughput screening, are reviewed in [10]. The engineering of a lysis system that is triggered by a user-defined signal can avoid the use of common cell disruption techniques for the recovery of intracellularly expressed proteins.

Another important application of an inducible lytic system is the programmed cell death of a bacterial population, which might be useful in those processes where the microorganism must be eliminated at a specific time, after having completed its work.

In literature, inducible lysis systems have been proposed. T4 phage holin and T7 phage lysozyme genes have been used to construct lytic E. coli strains to achieve the gentle disruption of cells upon IPTG induction using the lac promoter or the DE3 inducible system $[8,11]$. The T7 lysozyme was used to both cut bonds in the cell wall and tightly regulate holin gene by inhibiting the T7 polymerase basal expression in uninduced DE3 inducible system. The same genes have been used under the control of a glucose starvation-inducible promoter to allow cell autolysis upon glucose exhaustion in the medium [12]. Heat- and UV-inducible promoters have been used to regulate the lambda phage lysis cassette $S R R z$ for high throughput enzyme release [13].

The MIT Registry of Standard Biological Parts [14] hosts several lysis protein coding sequences and devices, but most of them, despite their important potential applications, remain uncharacterized. The BioBrick ${ }^{\mathrm{TM}}$ device ${ }^{1}$ BBa_K112808 consists of a promoterless operon composed by the T4 phage genes $t$ and $e$, encoding a holin and a lysozyme respectively [15]. Downstream of the transcriptional terminator of the operon, the T4 rI gene, encoding a $t$-specific antiholin, is present under the control of a weak constitutive promoter (see Figure $1 \mathrm{~A}$ for a detailed overview of this lysis device). The T4 antiholin is able to heterodimerize with the $t$ gene product, thus avoiding the holin to form pores in the inner membrane [16]. When the lysis device is assembled under the control of an inducible promoter, a weak constitutive expression of $r I$ prevents cell lysis, which may be caused by the basal expression of the promoter in the off-state, and in this way the tight regulation of the inducible lysis can be obtained.

In this work, BioBrick ${ }^{\mathrm{TM}}$ parts have been used to quantitatively characterize the BBa_K112808 lysis device by assembling it to BBa_F2620, a well characterized N3-oxohexanoyl-L-homoserine lactone (HSL)-inducible device based on the lux promoter (see Figure 1B for a working diagram of this device) [17], thus yielding the new BioBrick ${ }^{\mathrm{TM}}$ BBa_K173015. BBa_F2620, whose transfer function has already been measured, has been used to drive the expression of BBa_K112808 over a range of transcriptional input values and to characterize the lysis behavior as a function of the transcriptional strength in E. coli. Finally, in order to study the modularity of this device, a different inducible input device, which can be triggered by heat induction (see Figure $1 \mathrm{C}$ for a working diagram of this device), has been assembled upstream, thus yielding the new BioBrick ${ }^{\mathrm{TM}} \mathrm{BBa} J 107014$, and the validation of this composite part has been carried out. Figure 1D shows the maps of the three main plasmids used in this work, containing the promoterless lysis device (pLC-T4Lys ${ }^{-}$), the HSL-inducible lysis device (pLC-T4LysHSL) and the heat-inducible lysis device (pLC-T4LysHeat) respectively, all of them in a low copy number vector.

\section{Methods}

\section{Strains and plasmids}

All the E. coli strains and plasmids used in this study are listed in Table 1. The BioBrick ${ }^{\mathrm{TM}}$ codes reported in the table are given according to the Registry of Standard Biological Parts [14].

pSB1A2 and BBa_J61002 are high copy number vectors with a pUC19-derived pMB1 replication origin ( 100-300 molecules per cell) and Ampicillin resistance marker, while pSB4C5 is a low copy number vector with a pSC101 replication origin ( $\sim 5$ molecules per cell) and Chloramphenicol resistance marker [18]. The full description of vectors and inserts, including their nucleotide sequence, can be found in the BioBrick ${ }^{\mathrm{TM}}$ individual pages in the Registry of Standard Biological Parts web site.

\section{Cloning methods}

Chemically competent TOP10 E. coli (Invitrogen) were routinely used both for cloning and for quantitative experiments. Chemically competent DB3.1 E. coli (Invitrogen) were used to propagate $\mathrm{pLC}$-ccdB plasmid, containing a $\mathrm{ccdB}$ expression cassette, which is toxic for TOP10 but not for DB3.1. DH5alpha (Invitrogen) and MG1655 (purchased from CGSC, Yale University, USA) were only used for quantitative experiments. TOP10, DH5alpha and DB3.1 were heat shock-transformed according to the manufacturer's protocol. MG1655 were made chemically competent with the protocol described in [19] and they were heat-shock transformed at $42^{\circ} \mathrm{C}$ with the required plasmid. All the strains were routinely grown at $37^{\circ} \mathrm{C}$ in selective LB medium [19] with Ampicillin $(100 \mu \mathrm{g} / \mathrm{ml})$ or Chloramphenicol $(12.5 \mu \mathrm{g} / \mathrm{ml})$ to propagate plasmids, except pLC-T4LysHeat that was grown at $30^{\circ} \mathrm{C}$ to avoid heat-induction of lysis genes. 




IM

$(A)$
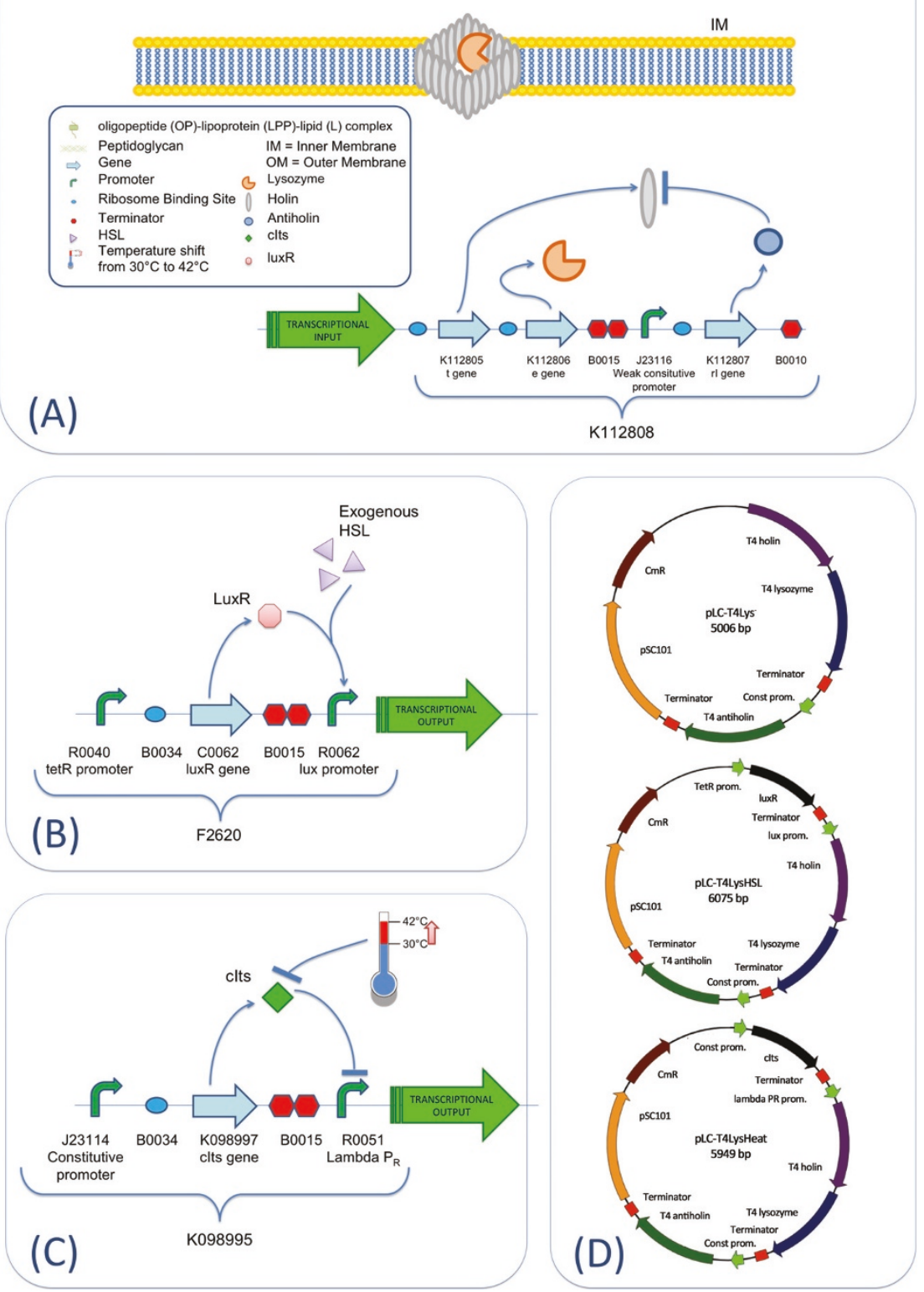

Figure 1 Overview of the devices used in this work. The gram-negative bacteria envelope consists in an inner membrane, an outer membrane and a peptidoglycan layer, which is linked to the outer membrane via oligopeptide (OP)-lipoprotein (LPP)-lipid (L) complexes and gives structural strength to the cell wall [25]. The lysis device is composed by a promoterless operon encoding a holin (gene $t$ ) and a lysozyme (gene e). When the operon is expressed, holin forms lesions in the inner membrane. Lysozyme can pass through these lesions, reaching and attacking the peptidoglycan layer, thus achieving cell lysis. In addition, the weak promoter BBa_J23116 constitutively expresses gene $r$ l, encoding an antiholin, which inhibits the holin action caused by basal expression of the t-e operon (A). BBa_F2620 HSL-inducible input device is composed by a luxR expression cassette, driven by the tetR promoter, and the lux promoter, which is normally off. Its transcription can be induced by the LuxR transcription factor in presence of exogenously added HSL (B). BBa_K098995 heat-inducible input device is composed by a clts expression cassette, driven by the BBa_J23114 constitutive promoter, and PR repressible promoter from lambda phage. clts is a heat-sensitive repressor of PR: when temperature is $30^{\circ} \mathrm{C}$ the repressor tightly keeps the promoter in the off-state, while a temperature shift to $42^{\circ} \mathrm{C}$ can induce the transcription of PR (C). Plasmid maps of the promoterless lysis device PLC-T4Lys', the HSL-inducible lysis device PLC-T4LysHSL and the heatinducible lysis device PLC-T4LysHeat, all of them in a low copy number vector (D). 
Table 1 Plasmids and strains used in this study

\begin{tabular}{|c|c|c|}
\hline \multicolumn{3}{|c|}{ Strains } \\
\hline Name & Genotype & Source \\
\hline TOP10 & $\begin{array}{c}\text { F- mcrA } \Delta(\text { mrr-hsdRMS-mcrBC) } \phi 80 \text { lacZ } \Delta M 15 \Delta \text { lacX74 nupG recA1 } \\
\left.\text { araD139 } \Delta \text { (ara-leu)7697 galE15 galK16 rpsL(Str }{ }^{R}\right) \text { endA1 } \lambda^{-}\end{array}$ & Invitrogen \\
\hline DB3.1 & 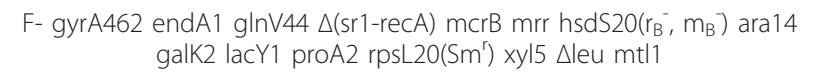 & Invitrogen \\
\hline DH5alpha & $\begin{array}{l}\text { F- endA1 glnV44 thi-1 recA1 relA1 gyrA96 deoR nupG } \phi 80 \text { dlacZ } \triangle M 15 \Delta \\
\text { (lacZYA-argF)U169, hsdR17(rK- mK+), } \lambda^{-}\end{array}$ & Invitrogen \\
\hline MG1655 & F- $\lambda$ - ilvG- rfb-50 rph-1 & CGSC \\
\hline
\end{tabular}

\begin{tabular}{|c|c|c|}
\hline & & \\
\hline Name & BioBrick ${ }^{\mathrm{TM}}$ code & Description \\
\hline $\mathrm{pLC}-\mathrm{ccdB}$ & pSB4C5(BBa_152002) ${ }^{a}$ & $\begin{array}{l}\text { pUC19-derived pMB1 replication origin and } c c d B \text { toxin } \\
\text { constitutive expression cassette in low copy vector }\end{array}$ \\
\hline $\begin{array}{l}\text { pHC- } \\
\text { T4Lys }\end{array}$ & pSB1A2(BBa_K112808) $)^{\mathrm{a}}$ & promoterless lysis device in high copy vector \\
\hline $\mathrm{pHC}-\mathrm{HSL}$ & pSB1A2(BBa_F2620) $)^{b}$ & HSL-inducible promoter in high copy vector \\
\hline pHC-RFP & BBa_J61002(BBa_J23118) ${ }^{a}$ & RFP constitutive expression cassette \\
\hline pHC-Heat & pSB1A2(BBa_K098995) $)^{\mathrm{a}}$ & heat-inducible promoter in high copy vector \\
\hline $\begin{array}{c}\text { pHC- } \\
\text { T4LysHSL }\end{array}$ & pSB1A2(BBa_K173015) ${ }^{c}$ & HSL-inducible lysis device in high copy vector \\
\hline $\mathrm{pLC}-\mathrm{HSL}$ & pSB4C5(BBa_F2620) $)^{c}$ & HSL-inducible promoter in low copy vector \\
\hline $\begin{array}{l}\text { pLC- } \\
\text { T4Lys }\end{array}$ & pSB4C5(BBa_K112808) ${ }^{\mathrm{C}}$ & promoterless lysis device in low copy vector \\
\hline $\begin{array}{l}\text { pLC- } \\
\text { T4LysHSL }\end{array}$ & pSB4C5(BBa_K173015) ${ }^{\mathrm{c}}$ & HSL-inducible lysis device in low copy vector \\
\hline $\begin{array}{l}\text { pLC- } \\
\text { T4LysHeat }\end{array}$ & pSB4C5(BBa_J107014) ${ }^{c}$ & heat-inducible lysis device in low copy vector \\
\hline
\end{tabular}

a taken from the Registry DNA Distribution 2009

${ }^{\mathrm{b}}$ given by the iGEM Headquarters, Massachusetts Institute of Technology, Cambridge, USA

c constructed in this study

The BioBrick ${ }^{\mathrm{TM}}$ code of the plasmids include the vector name (out of brackets) and insert name (in brackets). The prefix pHC- indicates a high copy vector backbone (pSB1A2 or BBa_J61002), while pLC- indicates a low copy vector backbone (pSB4C5).

Plasmids have been purified through QIAprep Spin Miniprep kit (Qiagen) from $5 \mathrm{ml}$ overnight cultures. For every plasmid, $750 \mu \mathrm{l}$ of culture were mixed with $250 \mu \mathrm{l}$ of sterile $80 \%$ glycerol solution for long-term storage at $-80^{\circ} \mathrm{C}$. DNA was digested with EcoRI, XbaI, SpeI or PstI according to BioBrick ${ }^{\mathrm{TM}}$ Standard Assembly procedure [20] and isolated from 1\% agarose gel by Gel Extraction Kit (Roche Diagnostics). Cloning of parts was assessed by $\mathrm{T} 4$ Ligase and ligation products were heated at $65^{\circ} \mathrm{C}$ for $10 \mathrm{~min}$ to inactivate $\mathrm{T} 4$ Ligase before proceeding with heat shock transformation. All the enzymes were purchased from Roche Diagnostics and used according to manufacturer's protocol.

TOP10-rfp-lys strain (see Protein release assays section), which bears two plasmids, pHC-RFP and pLCT4LysHSL, was built up with the following procedure: competent TOP10 were transformed with pLCT4LysHSL, then transformed cells were grown in selective LB and made chemically competent again with the protocol described in [19] and heat shock-transformed at $42^{\circ} \mathrm{C}$ with pHC-RFP. Co-transformants were selected using LB medium with Ampicillin at $100 \mathrm{ug} / \mathrm{ml}$ and Chloramphenicol at $12.5 \mathrm{ug} / \mathrm{ml}$.

LB and M9 supplemented medium (11.28 g/L M9 salts, $1 \mathrm{mM}$ thiamine hydrochloride, $2 \mathrm{mM}$ MgSO4, 0.1 $\mathrm{mM} \mathrm{CaCl} 2,0.2 \%$ casamino acids and $0.4 \% \mathrm{vol} / \mathrm{vol}$ glycerol as carbon source) [19] were used in quantitative experiments.

DNA sequencing was performed through BMR Genomics (Padova, Italy) DNA analysis service.

\section{Plasmid construction}

pSB4C5 low copy vector bulk, obtained from pLC-ccdB after EcoRI-PstI cut, was ligated to the inserts of pHCT4Lys ${ }^{-}$and pHC-HSL cut with EcoRI-PstI to yield pLC-T4Lys and pLC-HSL respectively. pHCT4LysHSL was constructed by assembling the insert of pHC-HSL cut with EcoRI-SpeI to pHC-T4Lys cut with EcoRI-XbaI. pLC-T4LysHSL was constructed by assembling the insert of pHC-T4Lys ${ }^{-}$cut with XbaIPstI to pLC-HSL cut with SpeI-PstI. Finally, pLCT4LysHeat was constructed by assembling the insert of 
pHC-Heat cut with EcoRI-SpeI to pLC-T4Lys ${ }^{-}$cut with EcoRI-XbaI.

\section{Lysis assays}

Unless otherwise noted, $5 \mu \mathrm{l}$ of bacteria bearing pLCT4LysHSL and pLC-T4Lys glycerol stocks were inoculated in $5 \mathrm{ml}$ of selective LB medium and grown at $37^{\circ}$ C, $220 \mathrm{rpm}$ overnight. The cultures were diluted 1:100 in $5 \mathrm{ml}$ of selective LB medium and grown for additional 4-5 hours under the same conditions as before. After that time, a $200 \mu \mathrm{l}$ aliquot of each culture was transferred in a flat-bottomed 96-well microplate (Greiner) and the OD600 was measured with an Infinite F200 microplate reader (Tecan). Based on this measurement, the cultures were diluted to the same OD600 (0.05-0.13) and then six $200 \mu \mathrm{l}$ aliquots of each culture were transferred in a flat-bottomed 96-well microplate (Greiner). Unless otherwise noted, three wells of each culture were induced with $2 \mu \mathrm{l}$ of properly diluted HSL (Sigma Aldrich \#K3007) and $2 \mu \mathrm{l}$ of deionized water were added to the other three wells (uninduced wells). If the HSL-inducible lysis device had to be assayed with different HSL concentrations in the same experiment, three $200 \mu \mathrm{l}$ aliquots of the cultures for each investigated concentration were transferred in the microplate and induced. The microplate was incubated at $37^{\circ} \mathrm{C}$ in the Infinite F200 microplate reader and assayed every 5 min following this protocol immediately before the measurement: $15 \mathrm{~s}$ of linear shaking (amplitude $=3 \mathrm{~mm}$ ), wait for $5 \mathrm{~s}$, OD600 measurement.

For lysis assays on pLC-T4LysHeat, the cultures were grown at $30^{\circ} \mathrm{C}$ instead of $37^{\circ} \mathrm{C}$ and an automatic temperature shift from $30^{\circ} \mathrm{C}$ to $42^{\circ} \mathrm{C}$ was used to induce lysis in the microplate reader instead of HSL.

\section{Analysis of growth curves}

Raw OD600 values measured in the Infinite F200 microplate reader were normalized by subtracting for each time point the mean raw absorbance of the media to compute the actual bacterial optical density.

The growth phases of bacterial cultures with the different plasmids used in this study, grown in LB medium in a microplate, have been characterized in each experiment by computing the natural logarithm of the OD600 values $\ln (O D 600(t))$ over time. Then, the exponential phase was identified by visual inspection as the linear region of $\ln (O D 600(t))$, the late stationary phase as the constant region and the early stationary phase as the region between the other two.

In all the lysis assays, uninduced bacteria doubling time in exponential growth phase was evaluated by performing linear regression over the $\ln (O D 600(t))$ linear region to estimate the curve slope $m$, which represents the growth rate of the culture. Then the culture doubling time was computed as $\ln (2) / m$.

The lysis entity after induction was computed as $100^{*}$ (1- $\min _{O D 600} /$ ref $_{O D 600}$ ), where $\min _{O D 600}$ is the minimum OD600 reached by the culture and $r e f_{O D 600}$ is the OD600 immediately before the density drop caused by cell lysis. The rise time was computed as the time required for the $100 *\left(1-O D 600(t) / r^{*} f O D_{600}\right)$ signal to rise from $10 \%$ to $90 \%$ of the lysis entity.

\section{Protein release assays}

$5 \mu \mathrm{l}$ of TOP10-rfp-lys, TOP10 bearing pHC-RFP and TOP10 bearing pLC-T4Lys ${ }^{-}$glycerol stocks were inoculated in $5 \mathrm{ml}$ of selective LB medium and grown at $37^{\circ}$ C, $220 \mathrm{rpm}$ overnight. TOP10-rfp-lys and TOP10 with pHC-RFP cultures were diluted 1:100 into six $15 \mathrm{ml}$ tubes containing $5 \mathrm{ml}$ of pre-warmed selective LB medium. TOP10 with pLC-T4Lys ${ }^{-}$culture was diluted 1:100 in one $15 \mathrm{ml}$ tube with $5 \mathrm{ml}$ of pre-warmed selective LB. The 13 resulting cultures were grown to an OD600 of about 0.55 (exponential phase). Three of the six replicates of TOP10-rfp-lys and TOP10 with pHC-RFP were induced with HSL at a final concentration of $100 \mathrm{nM}$ and all the 13 tubes were incubated under the same conditions as before for $125 \mathrm{~min}$. Every $25 \mathrm{~min}$, the OD600 was measured with the NanoDrop ND-1000 and a $300 \mu \mathrm{l}$ aliquot was taken from each culture. The 300 $\mu \mathrm{l}$ samples were centrifuged at $11000 \mathrm{rpm}$ in a table top centrifuge and the fluorescence of $200 \mu \mathrm{l}$ of the supernatant was measured in a microplate with the Infinite F200 reader using: $535 \mathrm{~nm}$ excitation filter, $620 \mathrm{~nm}$ emission filter, excitation bandwidth $=25 \mathrm{~nm}$, emission bandwidth $=20 \mathrm{~nm}$, gain $=55$, number of flashes $=25$, integration time $=20 \mu \mathrm{s}$, top reading. The raw fluorescence measurements of TOP10-rfp-lys and TOP10 bearing pHC-RFP (induced and uninduced) were normalized by subtracting the background fluorescence value of pLC-T4Lys ${ }^{-}$cells at the same time points.

To measure the percentage of the RFP released in the medium after 125 minutes, TOP10-rfp-lys, TOP10 bearing PHC-RFP and TOP10 bearing PLC-T4Lys ${ }^{-}$were grown and induced as described above, in $3 \mathrm{ml}$ cultures. At $\mathrm{t}=125 \mathrm{~min}$, the cultures were centrifuged (4000 $\mathrm{rpm}, 4^{\circ} \mathrm{C}$ for $10 \mathrm{~min}$ ) and supernatants and pellets were processed to measure the amount of intracellular and extracellular RFP. Pellets were resuspended in $200 \mu \mathrm{l}$ of sterile LB broth and $200 \mu \mathrm{l}$ of $2 \mathrm{X}$ lysis buffer $(25 \mathrm{mM}$ of Tris- $\mathrm{HCl}$ at $\mathrm{pH} 8.0,8 \%$ SDS) were added to the resuspended pellets [21]. The resuspended pellets with the lysis buffer were left at room temperature for $15 \mathrm{~min}$, then transferred in a $1.5 \mathrm{ml}$ tube and centrifuged at $13000 \mathrm{rpm}$ for $15 \mathrm{~min}$ in a table top centrifuge to spin down the cell debris. $200 \mu \mathrm{l}$ of these supernatants were transferred in a microplate and the RFP fluorescence 
was measured as described above, to evaluate the intracellular RFP amount. To evaluate the extracellular RFP concentration, the growth media were treated as follows: $100 \mu \mathrm{l}$ of media were mixed with $100 \mu \mathrm{l}$ of $2 \mathrm{X}$ lysis buffer, left at room temperature for $15 \mathrm{~min}$, transferred in a microplate and the fluorescence measured in the same way as the lysed pellets. The $2 \mathrm{X}$ lysis buffer was added to reproduce the same conditions as the pellets. The raw intracellular and extracellular RFP measurements were normalized by subtracting the background fluorescence of pLC-T4Lys ${ }^{-}$lysed pellet and supernatant respectively. Finally, the mean RFP values of lysed pellets and supernatants of TOP10-rfp-lys and TOP10 bearing pHC-RFP (induced and uninduced) were corrected by the total culture volume, so the percentage of extracellular RFP molecules was computed for each culture (see Additional file 1, Supplementary Methods and Supplementary Table 1 for details).

\section{Optical density calibration}

OD600 measurements performed with the Infinite F200 microplate reader and the NanoDrop ND-1000 were converted to OD600 measurements in $1 \mathrm{~cm}$ pathlength by calibrating the two instruments with the V-530 spectrophotometer (Jasco), measuring the OD600 of serial dilutions of a TOP10 culture grown in LB or M9 supplemented medium.

\section{Evolutionary stability characterization}

Bacteria bearing pLC-T4LysHSL were propagated for 100 generations as described in [22] without adding HSL and every 10 generations a lysis assay was performed to test the stability of the lysis phenotype. Stability was assessed by measuring lysis entity in each assay. In particular, in order to achieve 100 generations, the culture was diluted 1:1000 every 24 hours, thus yielding about 10 generations per day $\left(\log _{2} 1000=9.97\right)$ [22] Every day, an aliquot was taken from the propagated culture and lysis was assayed as described above. This experiment was performed in triplicate for each tested strain.

\section{Analysis of mutants}

To analyze mutants, a lysis assay was performed on TOP10 bearing pLC-T4LysHSL induced with HSL 10 $\mathrm{nM}$ and when the lysed and re-grown cells reached an OD600 $=0.22$ they were diluted 1:1000 in fresh selective LB medium in a new 96-well microplate, incubated in the Infinite F200 reader and induced again with HSL 10 $\mathrm{nM}$ when they reached an OD600 of 0.35 (exponential phase) to check if they could lyse again. The re-grown cells were also streaked on selective LB agar, then 2 single colonies were inoculated in $5 \mathrm{ml}$ of selective LB and let grow overnight $\left(37^{\circ} \mathrm{C}, 220 \mathrm{rpm}\right)$. Plasmid DNA was extracted from the $5 \mathrm{ml}$ overnight cultures and analyzed by restriction enzyme digestion/electrophoresis and DNA sequencing using primers VF2: 5'-TGCCACCT GACGTCTAAGAA-3', VR: 5'-ATTACCGCCTTT GAGTGAGC-3' and C0062VF: 5'-GAATGTTTAGC GTGGGCATG-3'. This procedure was carried out starting from $5 \mu \mathrm{l}$ of pLC-T4LysHSL glycerol stock and also from single colonies isolated from this stock.

\section{Results and Discussion}

\section{Lysis assays}

Lysis was assayed in a 96-well microplate by measuring the optical density at $600 \mathrm{~nm}$ (OD600) dynamics of TOP10 bearing HSL-inducible lysis device pLCT4LysHSL, induced with HSL and uninduced. Induced and uninduced TOP10 bearing the promoterless lysis device pLC-T4Lys ${ }^{-}$were used as a negative control in all the experiments. Lysis entity, i.e. the maximum percent reduction of OD600 caused by lysis induction, was computed in all the assays as an indirect measurement of the amount of lysed cells.

A typical lysis profile is reported in Figure 2, where TOP10 bearing pLC-T4LysHSL were induced with HSL $100 \mathrm{nM}$ at $\mathrm{t}=0 \mathrm{~h}(\mathrm{OD} 600=0.2$, exponential phase $), \mathrm{t}$ $=4 \mathrm{~h}($ OD600 1.3, early stationary phase $)$ or $\mathrm{t}=20 \mathrm{~h}$ (OD600 2, late stationary phase). Lysis began after about 15 min from the induction in all the growth phases and its mean entity was about $76.3 \pm 0.3 \%, 75.4$ $\pm 1.1 \%$ and $50 \pm 2.5 \%$ at $\mathrm{t}=0 \mathrm{~h}, \mathrm{t}=4 \mathrm{~h}$ and $\mathrm{t}=20 \mathrm{~h}$, respectively (Table 2).

The mean doubling time of uninduced TOP10 bearing pLC-T4LysHSL, evaluated on all the experiments, was $49.8 \pm 1.1 \mathrm{~min}$, while the doubling time of the uninduced negative control pLC-T4Lys ${ }^{-}$was $43 \pm 1.3 \mathrm{~min}$, thus demonstrating that the HSL-inducible lysis device gives a reasonably low metabolic burden and allows the cells to grow at a rate comparable to their negative control (Table 2). In all cases, after about 2-3 h from the induction, cells start growing again, suggesting the onset of mutants that have lost the inducible lysis phenotype. Figure 3A shows the measured transfer function of pLC-T4LysHSL induced at $\mathrm{t}=4 \mathrm{~h}(\mathrm{OD}=0.9$, early stationary phase) with different HSL concentrations. The rise time, i.e. the time required to rise from $10 \%$ to $90 \%$ of the lysis entity, and the delay time before the OD600 drop are also reported for each HSL concentration (Figure $3 \mathrm{~B}$ and $3 \mathrm{C}$ ). As these parameters show, the lysis dynamics is highly nonlinear, in fact the delay and rise times change as a function of the induction entity. In particular, the delay time is equal for all the HSL concentrations except for the smallest one $(0.1 \mathrm{nM})$, in which it is about 40 min longer than measured for the other concentrations. The rise time increases as a function of the HSL concentration, reaching its highest value 

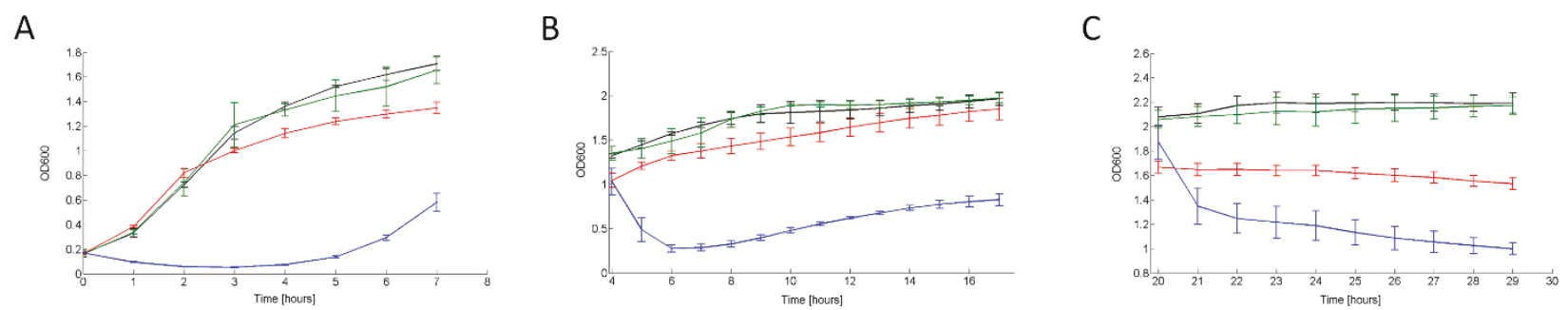

Figure 2 Lysis profile of TOP10 bearing the lysis device in low copy number when induced in different growth phases in microplate reader. OD600 of TOP10 with pLC-T4LysHSL induced with HSL 100 nM (blue line) and uninduced (red line), pLC-T4Lys induced with HSL 100 $\mathrm{nM}$ (green line) and uninduced (black line). Induction was performed in exponential phase (OD600 $=0.2)$ at $\mathrm{t}=0$ (A), early stationary phase (OD600 1.3) at $\mathrm{t}=4 \mathrm{~h}(\mathrm{~B})$ and late stationary phase (OD600 2) at $\mathrm{t}=20 \mathrm{~h}$ (C). Error bars represent the 95\% confidence interval of the estimated mean. For clarity of presentation, data points shown here are resampled with a 1-hour sampling time.

at $1 \mathrm{nM}$ HSL (more than 2.5 hours). However, at 0.1 $\mathrm{nM}$ the rise time is less than 1 hour.

Analogous studies were conducted for the HSL-inducible lysis device in high copy plasmid. However, for its evident instability and for the too high doubling time of uninduced cells (see Additional file 1, Supplementary Results for details), the lysis device in high copy plasmid has not been considered for further studies.

Lysis was also assayed for TOP10 bearing pLCT4LysHeat low copy vector containing a heat-inducible lysis device. Induction was triggered in a 96-well microplate by shifting the incubation temperature from $30^{\circ} \mathrm{C}$ to $42^{\circ} \mathrm{C}$. The results are shown in Figure 4 and summarized in Table 3 . The lysis entity was comparable to the one in the HSL-inducible device in low copy. The doubling time of the heat-inducible lysis device was similar to the negative control, but both were much higher than those reported in Table 2 because cultures were grown at $30^{\circ} \mathrm{C}$ instead of $37^{\circ} \mathrm{C}$, causing a slower growth rate. Also the time delay after induction in the thermoinducible lysis device is much higher than in the HSL-inducible device. This should be due to the different response time of the two input devices.

Experiments on pLC-T4LysHSL and pLC-T4LysHeat were also performed in a different growth medium (M9 with glycerol as carbon source, supplemented with thiamine and casamino acids) and in two other E. coli strains (DH5alpha and MG1655) grown in LB, giving consistent results when compared to the TOP10 strain in $\mathrm{LB}$ medium results described in this section (see Additional File 1, Supplementary Figure 1, Supplementary Figure 2 and Supplementary Table 3 for a detailed description of the results).

\section{Protein release assays}

A co-transformed TOP10 strain (here called TOP10-rfplys) bearing both a high copy plasmid with a Red Fluorescent Protein (RFP) constitutive expression cassette pHC-RFP and the HSL-inducible lysis device pLCT4LysHSL was induced with HSL to study the RFP release in the medium. Uninduced TOP10-rfp-lys, TOP10 bearing pHC-RFP (induced and uninduced) and TOP10 bearing pLC-T4Lys ${ }^{-}$were chosen as controls in this assay.

Figure $5 \mathrm{~A}$ and B show respectively the OD600 during the experiment and the fluorescence of the supernatant, which is proportional to the RFP molecules released in the growth medium. It is evident that only TOP10-rfplys + HSL $100 \mathrm{nM}$ could lyse and that cell lysis was accompanied by RFP release in the culture supernatant. For this culture, after 125 minutes from induction time, $96 \pm 0.04 \%$ of the RFP molecules had been released in the growth medium (see Table 4). Surprisingly, the three negative control cultures released about $25 \%$ of the molecules.

Table 2 Quantitative characterization of TOP10 bearing the HSL-inducible lysis device in low copy plasmid grown at $37^{\circ} \mathrm{C}$ in microplate

\begin{tabular}{cccc}
\hline & \multicolumn{2}{c}{ TOP10 with pLC-T4LysHSL } \\
\cline { 3 - 4 } & Exponential phase & Early stationary phase & Late stationary phase \\
\hline Lysis entity [\%] & $76.28 \pm 0.3$ & $75.43 \pm 1.1$ & $50.1 \pm 2.5$ \\
Lysis delay after induction [min] & 15 & 15 & 15 \\
Doubling time [min] & $49.8 \pm 1.1$ & & \\
Doubling time of negative control [min] & $43 \pm 1.3$ & \\
\hline
\end{tabular}

Induction was carried out with HSL $100 \mathrm{nM}$. Mean lysis entity and lysis delay after the induction are reported for the different growth phases together with their standard error, measured on 3 independent experiments. The doubling time of the uninduced lysis device and its negative control are reported too. 

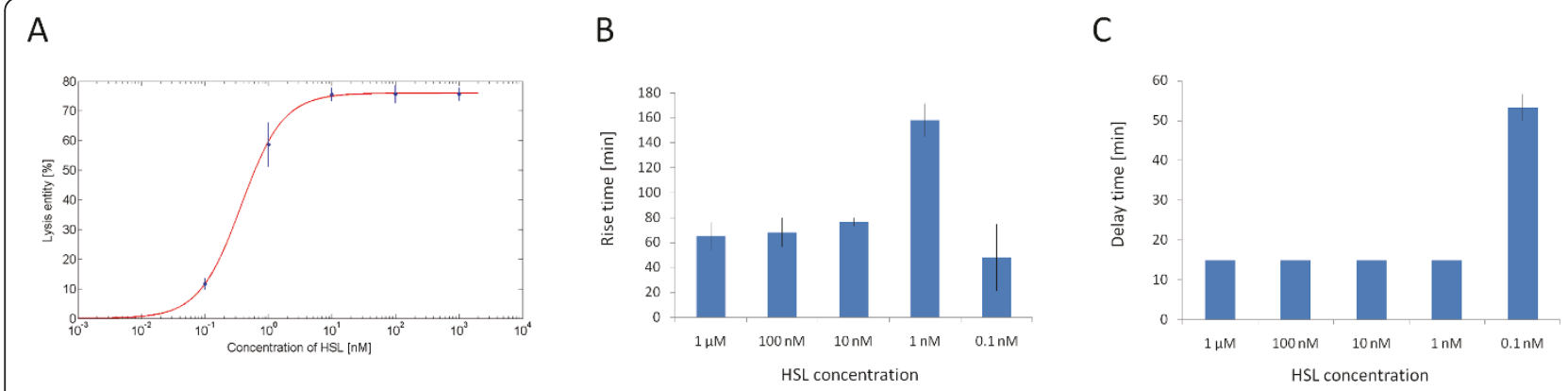

Figure 3 Transfer function, rise time and delay time of the HSL-inducible lysis device in low copy plasmid in early stationary phase in microplate reader. Lysis entity of TOP10 cells with pLC-T4LysHSL induced with different concentrations of HSL in early stationary phase at OD600 $=0.9$ (A). The experimental data (circles) were fitted with a Hill function (line, Vmax $=76, K_{50}=0.37, n=1.3$ ). For each concentration, the rise time, i.e. the time to rise from the $10 \%$ to $90 \%$ of the lysis entity (B) and the delay time before the OD600 drop after induction (C) are also shown. Error bars represent the $95 \%$ confidence interval of the estimated mean.

\section{Analysis of mutants}

After performing lysis assays, the lysed cultures grew again (see Lysis assays section). When the re-grown cultures of TOP10 bearing PLC-T4LysHSL were diluted 1:1000 in fresh selective LB medium and let grow to an OD600 $=0.35$ (exponential phase), they did not lyse upon induction with HSL $10 \mathrm{nM}$, suggesting that the cells have completely lost the inducible lysis phenotype (data not shown). The restriction analysis of the mutant plasmids after DNA digestion with EcoRI-PstI is reported in Figure 6. Plasmids were purified from two single colonies isolated from four mutant cultures. One of these cultures was obtained starting from $5 \mu \mathrm{l}$ of glycerol stock (here called mut gly $_{\text {culture), while the other }}$ three cultures were obtained starting from single colonies streaked from the glycerol stock (here called mut $_{\mathrm{sc} 1}$, mut $_{\mathrm{sc} 2}$ and mut $_{\mathrm{sc} 3}$ cultures respectively). In all

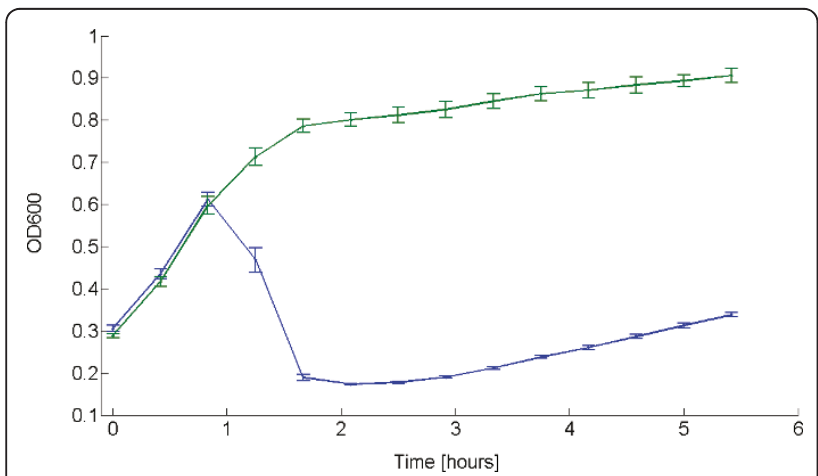

Figure 4 Lysis dynamics of TOP10 bearing the thermoinducible lysis device in low copy plasmid grown in microplate reader. OD600 of TOP10 with pLC-T4LysHeat induced with a temperature shift from $30^{\circ} \mathrm{C}$ to $42^{\circ} \mathrm{C}$ in the microplate reader (blue line). Heatinduced PLC-T4Lys (green line) is shown as the negative control. Induction was performed in exponential phase at $\mathrm{OD} 600=0.3$. Error bars represent the $95 \%$ confidence interval of the estimated mean. For clarity of presentation, data points shown here are resampled with a 30-minute sampling time. the mutant clones the pSB4C5 vector band ( 3.2 kbp) was present, but the insert was highly different from the unmutated culture ( 2.8 kbp), suggesting that mutations occurred just in the HSL-inducible lysis device. The sequencing of the clones disclosed all the mutations, as shown in Figure 7. Two clones (colony 2 of mut $_{\text {gly }}$ and colony 2 of mut $_{\mathrm{sc} 3}$ ) showed the insertion sequence IS10R after the nucleotide 576 of $\operatorname{luxR}$ (BBa_C0062) gene (Figure 7B); two clones (colony 2 of mut $\mathrm{sc}_{\mathrm{sc}}$ and colony 1 of mut $_{\mathrm{sc} 3}$ ) showed the insertion of IS10R in the same place as before, but in the opposite direction (Figure 7C); two clones (colony 1 and 2 of mut $_{\text {sc1 } 1}$ ) showed the deletion of the DNA comprising the lux promoter and the holin-lysozyme operon (Figure 7D); one clone (colony 1 of mut $_{\text {gly }}$ ) showed the insertion of IS10R after the nucleotide 318 of the holin (BBa_K112805) gene (Figure 7E); one clone (colony 1 of mut $_{\mathrm{sc} 2}$ ) had two different mutated plasmids in the same colony: one of them showed the insertion of IS10R before the nucleotide 1 of the luxR RBS (BBa_B0034), while the other one showed the insertion of IS5 before the start codon of luxR (Figure 7F). All the described mutations cause the impairment of the lysis phenotype. The molecular weight of the restriction fragments shown in Figure 6 are all consistent with the found mutations.

Table 3 Quantitative characterization of TOP10 bearing the thermoinducible lysis device in low copy plasmid grown at $30^{\circ} \mathrm{C}$ in microplate

\section{TOP10 with pLC-T4LysHeat}

\begin{tabular}{ll}
\hline Lysis entity in exponential phase [\%] & $73 \pm 0.3$ \\
Lysis delay after induction [min] & 55 \\
Doubling time [min] & $66.1 \pm 1.7$ \\
Doubling time of negative control [min] & $65.9 \pm 0.6$
\end{tabular}

Induction was carried out by shifting the temperature from $30^{\circ} \mathrm{C}$ to $42^{\circ} \mathrm{C}$. Mean values estimated on three wells in the same experiment are reported with standard errors. 


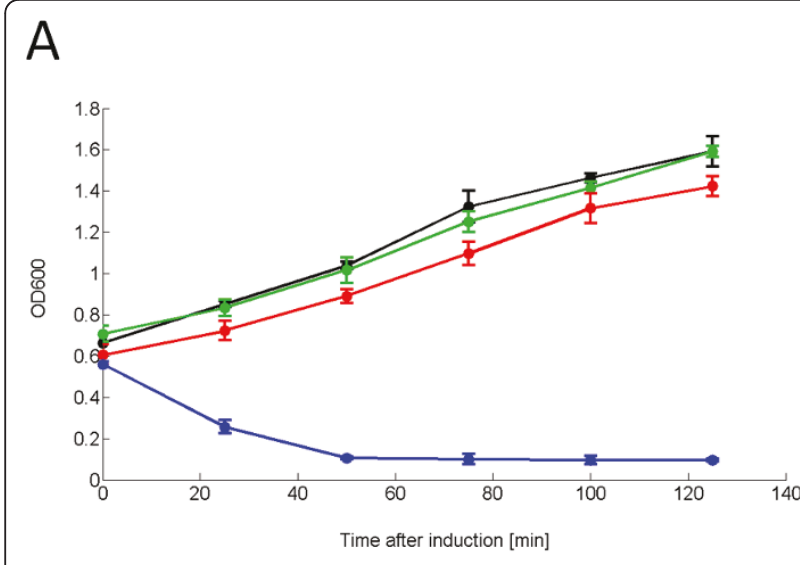

B

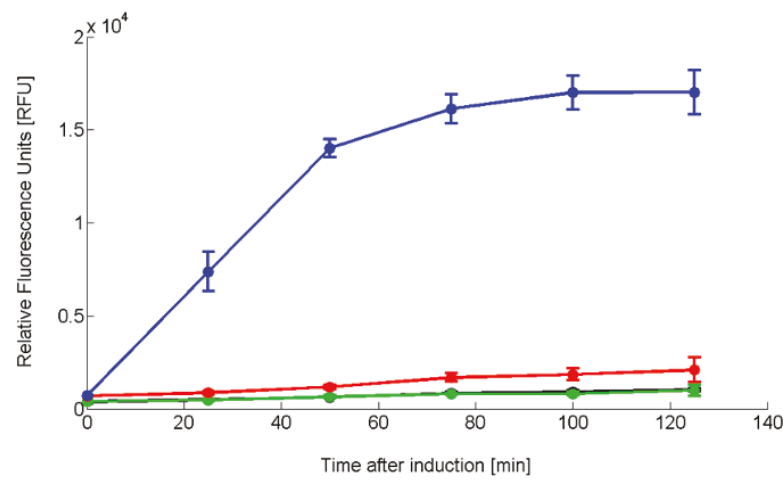

Figure 5 OD600 time course of TOP10-rfp-lys grown in $15 \mathrm{ml}$ tubes upon induction with HSL $100 \mathrm{nM}(\mathrm{A})$ and RFP fluorescence time course in the supernatant (B). Culture absorbance (A) and supernatant fluorescence (B) of TOP10-rfp-lys induced with HSL 100 nM (blue line). Uninduced TOP10-rfp-lys (red line), TOP10 bearing PHC-RFP induced with HSL $100 \mathrm{nM}$ (green line) or uninduced (black line) are the negative controls. Induction was carried out in the exponential phase at OD600 0.55. Error bars represent the $95 \%$ confidence interval of the estimated mean.

\section{Evolutionary stability of bacteria bearing pLC-T4LysHSL}

In order to study the reliability of the lysis device when cell disruption is not triggered, bacteria bearing pLCT4LysHSL were propagated for 100 generations without HSL and their inducible lysis phenotype was tested over time in terms of lysis entity. Supplementary Figure 3

Table 4 RFP release efficiency of TOP10-rfp-lys cultures grown at $37^{\circ} \mathrm{C}$ in $15 \mathrm{ml}$ tubes and induced with HSL $100 \mathrm{nM}$

\begin{tabular}{cc}
\hline Strain & Secreted RFP [\%] \\
\hline TOP10-rfp-lys & $24.84 \pm 0.6$ \\
TOP10-rfp-lys + HSL 100 nM & $95.95 \pm 0.04$ \\
TOP10 with pHC-RFP & $25.38 \pm 1.8$ \\
TOP10 with pHC-RFP + HSL 100 nM & $24.68 \pm 0.8$ \\
\hline
\end{tabular}

Mean values estimated on three tubes in the same experiment are reported with standard errors.

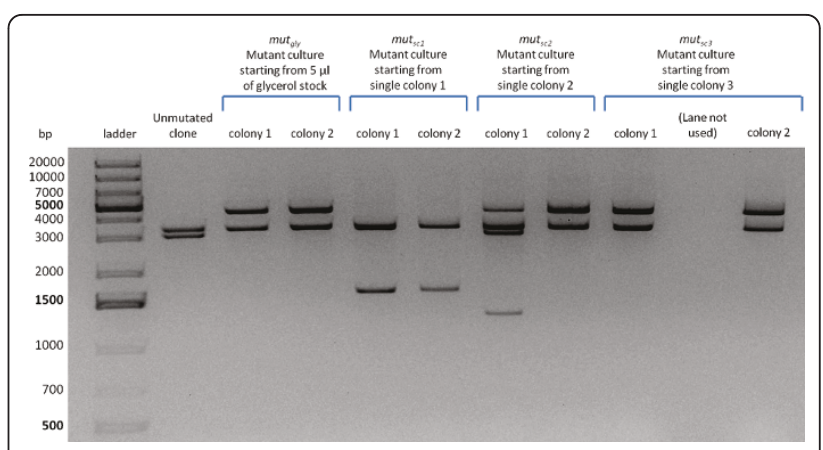

Figure 6 Restriction analysis of pLC-T4LysHSL mutants. Plasmid DNA was digested with EcoRI and Pstl. In all the screened clones two bands (vector backbone and insert) are present except in colony 1 of mut $_{s c 2}$, in which four bands can be observed. The expected vector backbone $(\sim 3.2 \mathrm{kbp})$ is present in all the clones, demonstrating that deletions or insertions occurred only in the insert, while all the mutated insert bands are clearly different from the unmutated culture ( $2.8 \mathrm{kbp})$. As sequencing showed, colony 1 of mut $_{s c 2}$ had plasmids with two different mutated inserts in the same clone, one of which containing an EcoRl restriction site that, when digested, produces 2 bands.

(Additional File 1) shows the results of this study in TOP10, DH5alpha and MG1655 grown in selective LB. Only MG1655 could maintain the phenotype of interest for 100 generations, thus showing an excellent stability of the uninduced lysis genes. Among these strains, TOP10 was the first to lose the lysis capability, which started to decrease after 30 generations and it was completely lost after 60. DH5alpha showed a high lysis entity variability because two of the three replicates completely lost the lysis capability after 60 generations, while the other one never lost it even in 100 generations, thus giving very wide standard errors of the measured mean.

\section{Conclusions}

In this work, the quantitative characterization of a BioBrick $^{\mathrm{TM}}$ lysis device of the Registry of Standard Biological Parts is reported. Its activity has been measured in E. coli using a well characterized HSL-inducible promoter and the transfer function, lysis dynamics, protein release capability, modularity and genotypic and phenotypic stability of the device have been evaluated.

Low copy number has been found to be the optimal working condition, as lysis could be triggered in all the growth phases of the bacterial culture and the cells grew with a relatively low metabolic burden, according to their doubling time. Lysis entity in late stationary phase was lower than in the other growth phases. These results are consistent with the published findings for which $E$. coli membrane disruption mediated by $\mathrm{T} 4$ phage lysis gene $t$ or $e$ was more efficient when the genes were expressed in the exponential growth phase than in the stationary phase, where less or almost no 


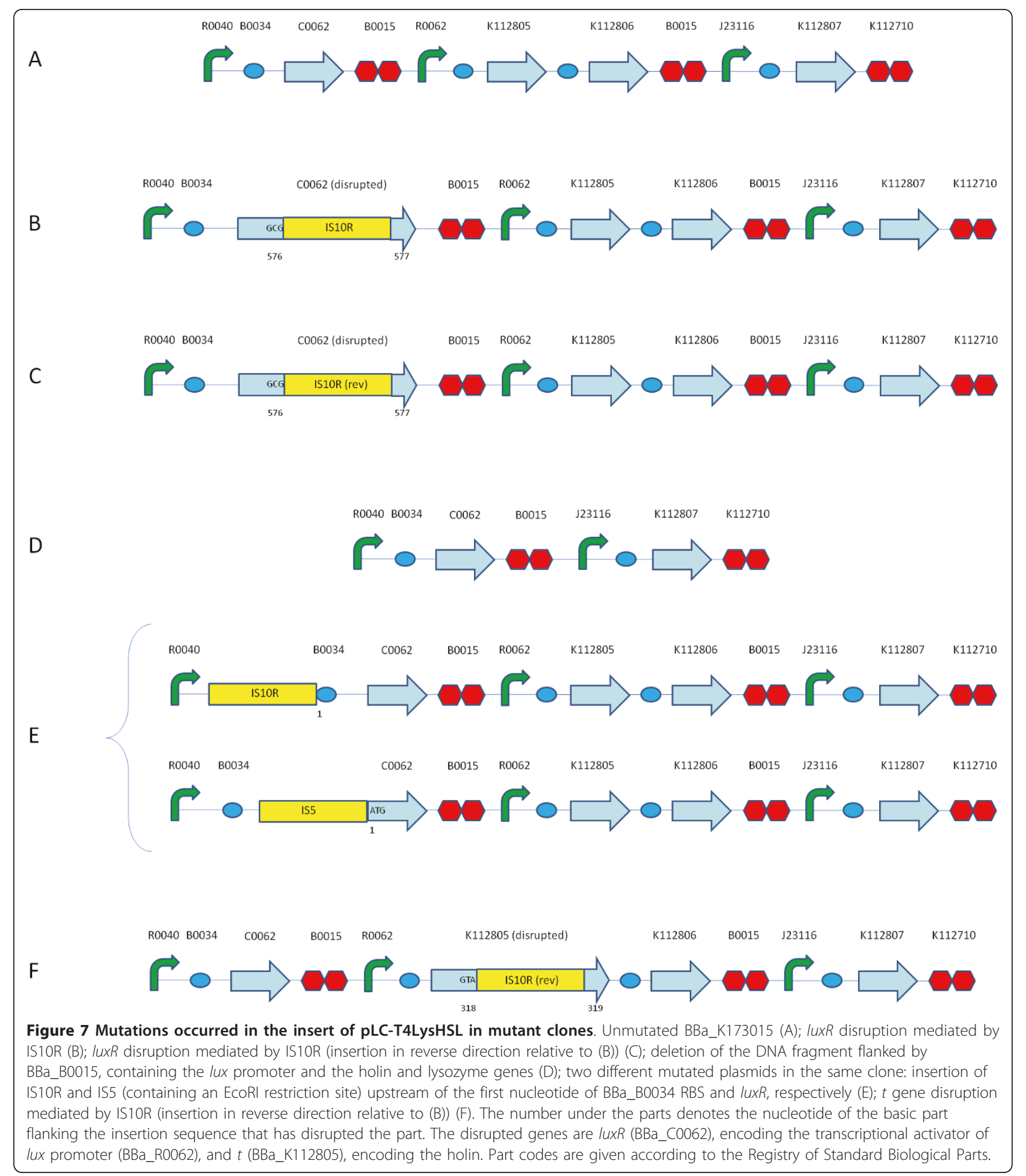

disruption occurred $[8,11,13]$. $96 \%$ of the total amount of intracellular proteins was successfully released into the growth medium upon induction of the lysis device in low copy plasmid. These features demonstrate that in this condition the expression of the lysis genes is tightly controlled and makes the device suitable for recombinant protein release upon gentle disruption of cell membranes. However, in all the experiments mutant cells that were unresponsive to induction arose after the bacterial lysis. This intrinsic instability makes the device 
unsuitable for the programmed cell death of a bacterial population. Mutant analysis showed that two main classes of DNA modifications occurred to eliminate the HSLinducible lysis phenotype. The most frequent one consisted in the insertion of IS10R or IS5 within the device sequence to impair the expression of the lysis genes by disrupting their regulatory parts upstream or the lysis genes themselves. The other class exhibited the deletion of genes included between two identical DNA sequences. In particular, the DNA fragment including the lux promoter and the holin and lysozyme genes was deleted from the plasmid most probably by a replication slippage mechanism between two identical transcriptional terminators (BBa_B0015) of 129 bp flanking the fragment. These mutations were consistent with published DNA mutations commonly occurring in E. coli [22], in fact gene disruptions mediated by insertion sequences, which occurred in the majority of the mutant cultures analyzed in this work, have been found to be responsible of $95 \%$ of the mutation events in TOP10 strain [23]. On the other hand, replication slippage events have already been found to occur between two BBa_B0015 terminators in a previously studied BioBrick ${ }^{\mathrm{TM}}$ device [24]. The HSL-inducible lysis device stability was also studied during continual bacterial growth for 100 generations without induction. The inducible lysis phenotype started decreasing after only 30 generations and was completely lost after 60, but DH5alpha and MG1655 strains gave a better performance. In particular, DH5alpha showed a significantly lower lysis capability after 60 generations, while MG1655 stably maintained it during all the 100 generations. The described lysis device has been shown to be compatible with other modular input devices. When assembled with a heat-inducible BioBrick $^{\mathrm{TM}}$ device upstream and triggered with a temperature shift to $42^{\circ} \mathrm{C}$, the lysis device worked as expected, thus demonstrating the possibility of triggering cell lysis with any transcription-based input device. This feature enables the intriguing possibility to control cell disruption in response to a user-defined exogenous signal.

All the results have been confirmed in different $E$. coli strains and different growth media, thus providing parameters that can be used in models to aid future biological systems design and to facilitate the re-usability of this lysis device.

The lysis device in high copy number plasmid gave worse performance, in fact lysis entity was lower than in low copy plasmid, the metabolic burden was much higher and the device was strongly unstable, as cell lysis induction usually failed to occur.

\section{Endnotes}

${ }^{1}$ BioBrick ${ }^{\mathrm{TM}}$ is a trademark of The BioBricks Foundation. (http://www.biobricks.org)

\section{Additional material}

Additional file 1: Supplementary Material, Figures and Tables

\section{Acknowledgements}

We thank Nicolò Politi for his help during quantitative experiments. This project was partially funded by the Italian "Ministero dell'Università e della Ricerca" through FIRB ITALBIONET and FIRB RBIP06FH7J projects.

\section{Author details}

'Dipartimento di Informatica e Sistemistica, Università degli Studi di Pavia, via Ferrata 1, Pavia, Italy. ${ }^{2}$ Centro di Ingegneria Tissutale, Università degli Studi di Pavia, via Ferrata 1, Pavia, Italy.

\section{Authors' contributions}

$L P, S Z, M L$ and PM designed the experiments. $L P, S Z$ and $M L$ performed all the plasmid constructions and validation experiments. LP, SZ and PM analyzed the data. LP, MGCDA and PM wrote the manuscript. All authors read and approved the final manuscript.

\section{Competing interests}

The authors declare that they have no competing interests.

Received: 28 November 2010 Accepted: 7 June 2011

Published: 7 June 2011

\section{References}

1. Young R: Bacteriophage lysis: mechanism and regulation. Microbio/ Rev 1992, 56:430-481.

2. Young R: Bacteriophage Holins: Deadly Diversity. J Mol Microbiol Biotechnol 2002, 4(1):21-36.

3. Tran TAT, Struck DK, Young R: Periplasmic Domains Define Holin-Antiholin Interactions in T4 Lysis Inhibition. Journal of Bacteriology 2005, 187(19):6631-6640.

4. Delisle AL, Barcak GJ, Guo M: Isolation and Expression of the Lysis Genes of Actinomyces naeslundii Phage Av-1. Applied and Environmental Microbiology 2006, 72(2):1110-1117.

5. Wang IN, Smith DL, Young R: HOLINS: The Protein Clocks of Bacteriophage Infections. Annual Review of Microbiology 2000, 54:799-825.

6. Gründling A, Manson MD, Young R: Holins kill without warning. Proc Natl Acad Sci USA 2001, 98(16):9348-9352.

7. Calendar R: The Bacteriophages Oxford University Press US; 2006.

8. Morita M, Asami K, Tanji Y, Unno H: Programmed Escherichia coli Cell Lysis by Expression of Cloned T4 Phage Lysis Genes. Biotechnology Progress 2001, 17(3):573-576.

9. Mergulhao FJM, Summers DK, Monteiro GA: Recombinant protein secretion in Escherichia coli. Biotechnology Advances 2005, 23:177-202.

10. Lin Z, Cai Z Dr: Cell lysis methods for high-throughput screening or miniaturized assays. Biotechnology Journal 2009, 4(2):210-215.

11. Tanji $Y$, Asami $K$, Xing $X$, Unno H: Controlled expression of lysis genes encoded in T4 phage for the gentle disruption of Escherichia coli cells. Journal of Fermentation and Bioengineering 1998, 85(1):74-78.

12. Yun J, Park J, Park N, Kang S, Ryu S: Development of a Novel Vector System for Programmed Cell Lysis in Escherichia coli. Journal of Microbiology and Biotechnology 2007, 17(7):1162-1168.

13. Cai Z, Xu W, Xue R, Lin Z, Facile : Reagentless and in situ release of Escherichia coli intracellular enzymes by heat-inducible autolytic vector for high-throughput screening. Protein Engineering, Design and Selection 2008, 21(11):681-687

14. MIT: Registry of Standard Biological Parts.[http://partsregistry.org].

15. UC Berkeley iGEM Team: Clonebots. 2008 [http://2008.igem.org/Team: UC_Berkeley].

16. Ramanculov E, Young R: An ancient player unmasked: T4 rl encodes a tspecific antiholin. Molecular Microbiology 2001, 41(3):575-583.

17. Canton B, Labno A, Endy D: Refinement and standardization of synthetic biological parts and devices. Nature Biotechnology 2008, 26:787-793.

18. Shetty R, Endy D, Knight T: Engineering BioBrick vectors from BioBrick parts. Journal of Biological Engineering 2008, 2:5. 
19. Sambrook J, Fritsch EF, Maniatis T: Molecular cloning: a laboratory manual Cold Spring Harbor Laboratory Press, Cold Spring Harbor, N.Y; 1989.

20. Knight T: Idempotent Vector Design for Standard Assembly of Biobricks. MIT DSpace 2003 [http://web.mit.edu/synbio/release/docs/biobricks.pdf].

21. Kumar JK, Tabor S, Richardson CC: Proteomic analysis of thioredoxintargeted proteins in Escherichia coli. PNAS 2004, 101(11):3759-3764.

22. Sleight SC, Bartley BA, Lieviant JA, Sauro HM: Designing and engineering evolutionary robust genetic circuits. Journal of Biological Engineering 2010, 4:12

23. Durfee T, Nelson R, Baldwin S, Plunkett G, Burland V, Mau B, Petrosino JF, Qin X, Muzny DM, Ayele M, Gibbs RA, Csörgo B, Pósfai G, Weinstock GM, Blattner FR: The Complete Genome Sequence of Escherichia coli DH10B: Insights into the Biology of a Laboratory Workhorse. J Bacteriol. 2008, 190(7):2597-2606.

24. Canton B: Engineering the interface between cellular chassis and synthetic biological systems. PhD Thesis, MIT 2008.

25. Young R, Wang IN, Roof WD: Phages will out: strategies of host cell lysis. Trends in Microbiology 2000, 8(3):120-128.

doi:10.1186/1754-1611-5-8

Cite this article as: Pasotti et al.: Characterization of a synthetic bacterial self-destruction device for programmed cell death and for recombinant proteins release. Journal of Biological Engineering 2011 5:8.

\section{Submit your next manuscript to BioMed Central} and take full advantage of:

- Convenient online submission

- Thorough peer review

- No space constraints or color figure charges

- Immediate publication on acceptance

- Inclusion in PubMed, CAS, Scopus and Google Scholar

- Research which is freely available for redistribution

Submit your manuscript at www.biomedcentral.com/submit 